\title{
Reicht eine antidepressive Monotherapie aus?
}

Fragestellung: Ist der Rückfallschutz nach einer erfolgreichen Elektrokrampftherapie (EKT) einerseits mit einer Monotherapie Escitalopram dosisabhängig erreichbar und andererseits mit dem einer Monotherapie mit Nortriptylin vergleichbar?

Hintergrund: Depressionen sollen bei besonderer Schwere der Erkrankung oder bei medikamentöser Therapieresistenz - auch nach der neuen nationalen Versorgungsleitlinie in Deutschland - mittels EKT behandelt werden. Eine klinisch sehr wichtige Frage stellt sich nach erfolgreicher EKT-Therapie: Wie kann man medikamentös zur Erhaltung des Therapieerfolgs beitragen?

Martiny K, Larsen ER, Licht RW et al. Relapse prevention in major depressive disorder after successful acute electroconvulsive treatment: a 6-month double-blind comparison of three fixed dosages of escitalopram and a fixed dose of nortriptyline - lessons from a failed randomised trial of the Danish University Antidepressant Group (DUAG-7). Pharmacopsychiatry 2015; $48: 274-8$
Patienten und Methodik: Diese Fragestellung sollte mittels einer doppelblinden, randomisierten und multizentrischen Studie beantwortet werden. Nach erfolgreicher EKT wurden 240 Patienten in vier Gruppen randomisiert: Laut Protokoll waren je 60 Patienten für eine Monotherapie von $10 \mathrm{mg}, 20 \mathrm{mg}$ oder $30 \mathrm{mg}$ Escitalopram oder $100 \mathrm{mg}$ Nortriptylin über 25 Wochen vorgesehen.
Es wurden nur Patienten mit unipolarer Depression und einem Post-EKT-Wert von $\leq 9$ Punkten in der Hamilton Depressionsskala (HAMD-17) eingeschlossen, was einer Remission nach der EKT-Behandlung entspricht. Um eine Monotherapie zu erreichen, wurden zudem innerhalb der ersten acht Wochen post EKT alle anderen Psychopharmaka ausgeschlichen, gleichzeitig wurde die Studienmedikation innerhalb einer Woche aufdosiert.

Ergebnisse: In die Studie wurden nur 47 von zirka 600 voruntersuchten Patienten eingeschlossen. 15 Patienten beendeten die Studie, 16 zeigten zuvor einen Rückfall, ein Patient beendete die Studie vor der ersten Medikamentengabe und 15 beendeten die Studie aufgrund von Nebenwirkungen (ein Patient), zu hohen Medikamentenspiegeln (drei Patienten) oder aus anderen Gründen (elf Patienten). Wahrscheinlich aufgrund der niedrigen Fallzahlen zeigten sich keine signifikanten Unterschiede für die drei verschiedenen Escitalopram-Dosen. Die zusammengefassten Escitalopram- versus Nortiptylin-Gaben zeigen in der KaplanMeier-Überlebenskurve keinen signifikanten Unterschied, aber einen Trend zugunsten der Nortriptylin-Gabe $(\mathrm{p}=0,08)$.

Schlussfolgerungen: Nur ein Drittel der eingeschlossenen Patienten war am Ende der Studie rückfallfrei, ein weiteres Drittel erkrankte erneut an einer depressiven Episode. Es zeigte sich weder eine Dosisabhängigkeit der Escitalopram-Gabe, noch ein Unterschied zur Nortriptylin-Gabe, wobei letzteres immerhin statistisches Trendniveau erreichte.

\section{- Kommentar von Alexander Sartorius, Mannheim}

\section{Medikamentöse Monotherapie nach EKT könnte zu kurz greifen}

Für den klinischen Alltag ist die in der Studie gestellte Frage von außerordentlichem Interesse. Bisher gibt es zu diesem Thema nur eine ähnliche Studie, die dreiarmig, doppelt verblindet und randomisiert Placebo gegen Nortriptylin und gegen Nortripylin plus Lithium verglichen hat [1]. Leider sind weder Lithium noch Nortriptylin immer klinisch gut verträglich und gerade Nortriptylin ist bei geriatrischen Patienten nicht nur wegen kardialer und kognitiver Nebenwirkungen problematisch. Es ist dennoch ein berichtenswertes Ergebnis, dass trotz niedriger Fallzahlen die Rückfallrate unter den Monotherapien der jetzigen Studie fast identisch ist mit den Ergebnissen des Monotherapiearms der Sackeim-Studie aus dem Jahr 2001. So gesehen ist das vermutete ungute Gefühl der Patienten, sich in eine Monotherapiestudie einschließen zu lassen, wohl nicht ganz ungerechtfertigt gewesen, genau wie das vermutete ungute Gefühl der behandelnde Psychiater, die diesbezüglich auch auf ihren eigenen Erfahrungsschatz zurückgreifen konnten. Auch die Autoren fordern aus diesem letztendlich misslungenen Studiendesign zu lernen: Künftige Studien sollten das Ausschleichen parallel gegebener Medikation entweder zulassen (was wirklichkeitsnäher wäre) oder vor der EKT-Serie beginnen. Zudem wären Vergleiche zwischen einem SSRI plus Lithium (als modernere Augmentationsstrategie) versus SSRI plus Mirtazapin (als Kombinationstherapie) viele näher an der derzeitigen klinischen Realität und würden so zu mehr klinisch relevanter Evidenz beitragen.

\author{
Referenz: \\ 1. Sackeim HA et al. JAMA 2001; 285: 1299-307
}

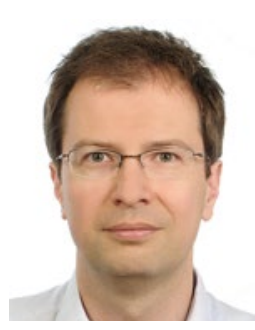

Prof. Dr. med. Dipl. Phys. Alexander Sartorius, Mannheim

Klinik für Psychiatrie und Psychotherapie, Zentralinstitut für Seelische Gesundheit, Medizinische Fakultät Mannheim/ Universität Heidelberg E-Mail: alexander.sartorius@zi-mannheim.de 